\author{
SEVER S. DRAGOMIR
}

\title{
Additive inequalities for weighted harmonic and arithmetic operator means
}

\begin{abstract}
In this paper we establish some new upper and lower bounds for the difference between the weighted arithmetic and harmonic operator means under various assumptions for the positive invertible operators $A, B$. Some applications when $A, B$ are bounded above and below by positive constants are given as well.
\end{abstract}

1. Introduction. Throughout this paper $A, B$ are positive invertible operators on a complex Hilbert space $(H,\langle\cdot, \cdot\rangle)$. We use the following notations for operators

$$
A \nabla_{\nu} B:=(1-\nu) A+\nu B
$$

the weighted operator arithmetic mean,

$$
A \sharp_{\nu} B:=A^{1 / 2}\left(A^{-1 / 2} B A^{-1 / 2}\right)^{\nu} A^{1 / 2},
$$

the weighted operator geometric mean and

$$
A !_{\nu} B:=\left((1-\nu) A^{-1}+\nu B^{-1}\right)^{-1},
$$

the weighted operator harmonic mean, where $\nu \in[0,1]$.

When $\nu=\frac{1}{2}$, we write $A \nabla B, A \sharp B$ and $A ! B$ for brevity, respectively.

2010 Mathematics Subject Classification. 47A63, 47A30, 15A60, 26D15, 26D10.

Key words and phrases. Young's inequality, convex functions, arithmetic meanharmonic mean inequality, operator means, operator inequalities. 
The following fundamental inequalities between the weighted arithmetic, geometric and harmonic operator means hold

$$
A{ }_{\nu} B \leq A \sharp_{\nu} B \leq A \nabla_{\nu} B
$$

for any $\nu \in[0,1]$.

For various recent inequalities between these means we recommend the recent papers $[3-6],[8-12]$ and the references therein.

In the recent work [7] we obtained, between others, the following result:

Theorem 1. Let $A, B$ be positive invertible operators and $M>m>0$ such that

$$
M A \geq B \geq m A .
$$

Then for any $\nu \in[0,1]$ we have

$$
r k(m, M) A \leq A \nabla_{\nu} B-A !_{\nu} B \leq R K(m, M) A,
$$

where $r=\min \{\nu, 1-\nu\}, R=\max \{\nu, 1-\nu\}$ and the quantities $K(m, M)$ and $k(m, M)$ are given by

$$
K(m, M):= \begin{cases}\frac{(m-1)^{2}}{m+1} & \text { if } M<1, \\ \max \left\{\frac{(m-1)^{2}}{m+1}, \frac{(M-1)^{2}}{M+1}\right\} & \text { if } m \leq 1 \leq M, \\ \frac{(M-1)^{2}}{M+1} & \text { if } 1<m\end{cases}
$$

and

$$
k(m, M):= \begin{cases}\frac{(M-1)^{2}}{M+1} & \text { if } M<1, \\ 0 & \text { if } m \leq 1 \leq M, \\ \frac{(m-1)^{2}}{m+1} & \text { if } 1<m .\end{cases}
$$

In particular,

$$
\frac{1}{2} k(m, M) A \leq A \nabla B-A ! B \leq \frac{1}{2} K(m, M) A .
$$

Let $A, B$ be positive invertible operators and positive real numbers $m$, $m^{\prime}, M, M^{\prime}$ such that the condition $0<m I \leq A \leq m^{\prime} I<M^{\prime} I \leq B \leq M I$ holds. Put $h:=\frac{M}{m}$ and $h^{\prime}:=\frac{M^{\prime}}{m^{\prime}}$, then for any $\nu \in[0,1]$ we have [7],

$$
r\left(h^{\prime}-1\right)^{2}\left(h^{\prime}+1\right)^{-1} A \leq A \nabla_{\nu} B-A{ }_{\nu} B \leq R(h-1)^{2}(h+1)^{-1} A,
$$

where $r=\min \{\nu, 1-\nu\}, R=\max \{\nu, 1-\nu\}$ and, in particular,

$$
\frac{1}{2}\left(h^{\prime}-1\right)^{2}\left(h^{\prime}+1\right)^{-1} A \leq A \nabla B-A ! B \leq \frac{1}{2}(h-1)^{2}(h+1)^{-1} A .
$$


Let $A, B$ be positive invertible operators and positive real numbers $m$, $m^{\prime}, M, M^{\prime}$ such that the condition $0<m I \leq B \leq m^{\prime} I<M^{\prime} I \leq A \leq M I$ holds. Then for any $\nu \in[0,1]$ we also have [7],

$$
\begin{aligned}
r\left(h^{\prime}-1\right)^{2}\left(h^{\prime}+1\right)^{-1}\left(h^{\prime}\right)^{-1} A & \leq A \nabla_{\nu} B-A !_{\nu} B \\
& \leq R(h-1)^{2}(h+1)^{-1} h^{-1} A
\end{aligned}
$$

and, in particular,

$$
\begin{aligned}
\frac{1}{2}\left(h^{\prime}-1\right)^{2}\left(h^{\prime}+1\right)^{-1}\left(h^{\prime}\right)^{-1} A & \leq A \nabla B-A ! B \\
& \leq \frac{1}{2}(h-1)^{2}(h+1)^{-1} h^{-1} A .
\end{aligned}
$$

Motivated by the above facts, in this paper we establish some new upper and lower bounds for the difference $A \nabla_{\nu} B-A !_{\nu} B$ for $\nu \in[0,1]$ under various assumptions for the positive invertible operators $A, B$. Some applications when $A, B$ are bounded above and below by positive constants are given as well.

2. Main results. We have:

Theorem 2. Let $A, B$ be positive invertible operators and $M>m>0$ such that the condition (1.2) is valid. Then for any $\nu \in[0,1]$ we have

$$
\begin{aligned}
& \nu(1-\nu) \min \left\{1, m^{3}\right\}\left(A B^{-1}-I\right)^{2} A \leq A \nabla_{\nu} B-A !_{\nu} B \\
& \leq \nu(1-\nu) \max \left\{1, M^{3}\right\}\left(A B^{-1}-I\right)^{2} A .
\end{aligned}
$$

In particular, we have

$$
\begin{aligned}
\frac{1}{4} \min \left\{1, m^{3}\right\}\left(A B^{-1}-I\right)^{2} A & \leq A \nabla B-A ! B \\
& \leq \frac{1}{4} \max \left\{1, M^{3}\right\}\left(A B^{-1}-I\right)^{2} A .
\end{aligned}
$$

Proof. Let $f: I \subset \mathbb{R} \rightarrow \mathbb{R}$ be a twice differentiable function on the interval $I$, the interior of $I$. If there exist constants $d, D$ such that

$$
d \leq f^{\prime \prime}(t) \leq D \text { for any } t \in \stackrel{\circ}{I}
$$

then [3],

$$
\begin{aligned}
\frac{1}{2} \nu(1-\nu) d(b-a)^{2} & \leq(1-\nu) f(a)+\nu f(b)-f((1-\nu) a+\nu b) \\
& \leq \frac{1}{2} \nu(1-\nu) D(b-a)^{2}
\end{aligned}
$$

for any $a, b \in \stackrel{\circ}{I}$ and $\nu \in[0,1]$.

Let $f:(0, \infty) \rightarrow(0, \infty)$ with $f(t)=\frac{1}{t}$. Then $f^{\prime \prime}(t)=\frac{2}{t^{3}}$ and if $t \in$ $[\min \{a, b\}, \max \{a, b\}]$, where $a, b>0$, then we have

$$
\frac{2}{\max ^{3}\{a, b\}} \leq f^{\prime \prime}(t) \leq \frac{2}{\min ^{3}\{a, b\}} .
$$


Using the inequality (2.4), we obtain

$$
\begin{aligned}
\nu(1-\nu) \frac{(b-a)^{2}}{\max ^{3}\{a, b\}} & \leq(1-\nu) \frac{1}{a}+\nu \frac{1}{b}-((1-\nu) a+\nu b)^{-1} \\
& \leq \nu(1-\nu) \frac{(b-a)^{2}}{\min ^{3}\{a, b\}}
\end{aligned}
$$

for any $a, b>0$ and $\nu \in[0,1]$.

If we take $a=\frac{1}{x}$ and $b=\frac{1}{y}$ in (2.5) with $x, y>0$, then we get

$$
\begin{aligned}
\nu(1-\nu) & (\min \{x, y\})^{3} \frac{(x-y)^{2}}{x^{2} y^{2}} \\
& \leq(1-\nu) x+\nu y-\left((1-\nu) x^{-1}+\nu y^{-1}\right)^{-1} \\
& \leq \nu(1-\nu)(\max \{x, y\})^{3} \frac{(x-y)^{2}}{x^{2} y^{2}} .
\end{aligned}
$$

Observe that

$$
\begin{aligned}
(\max \{x, y\})^{3} \frac{(x-y)^{2}}{x^{2} y^{2}} & =(\max \{x, y\})^{3} \frac{(x-y)^{2}}{(\max \{x, y\} \min \{x, y\})^{2}} \\
& =\max \{x, y\} \frac{(x-y)^{2}}{(\min \{x, y\})^{2}} \\
& =\max \{x, y\}\left(\frac{\max \{x, y\}}{\min \{x, y\}}-1\right)^{2}
\end{aligned}
$$

and, similarly

$$
(\min \{x, y\})^{3} \frac{(x-y)^{2}}{x^{2} y^{2}}=\min \{x, y\}\left(1-\frac{\min \{x, y\}}{\max \{x, y\}}\right)^{2}
$$

for any $x, y>0$.

Thus (2.6) is equivalent to

$$
\begin{aligned}
\nu(1-\nu) \min \{x, y\} & \left(1-\frac{\min \{x, y\}}{\max \{x, y\}}\right)^{2} \\
& \leq(1-\nu) x+\nu y-\left((1-\nu) x^{-1}+\nu y^{-1}\right)^{-1} \\
& \leq \nu(1-\nu) \max \{x, y\}\left(\frac{\max \{x, y\}}{\min \{x, y\}}-1\right)^{2}
\end{aligned}
$$

for any $x, y>0$ and $\nu \in[0,1]$.

Now, if we take $x=1$ in (2.6), then we get

$$
\begin{aligned}
\nu(1-\nu)(\min \{1, y\})^{3} & \left(y^{-1}-1\right)^{2} \\
& \leq 1-\nu+\nu y-\left(1-\nu+\nu y^{-1}\right)^{-1} \\
& \leq \nu(1-\nu)(\max \{1, y\})^{3}\left(y^{-1}-1\right)^{2}
\end{aligned}
$$


for any $y>0$ and $\nu \in[0,1]$.

If $y \in[m, M]$, then $\min \{1, y\} \geq \min \{1, m\}$ and $\max \{1, y\} \leq \max \{1, M\}$ and by (2.8) we get

$$
\begin{aligned}
\nu(1-\nu) \min \left\{1, m^{3}\right\} & \left(y^{-1}-1\right)^{2} \\
& \leq 1-\nu+\nu y-\left(1-\nu+\nu y^{-1}\right)^{-1} \\
& \leq \nu(1-\nu) \max \left\{1, M^{3}\right\}\left(y^{-1}-1\right)^{2}
\end{aligned}
$$

for any $y \in[m, M]$ and $\nu \in[0,1]$.

If we use the continuous functional calculus for the positive invertible operator $X$ with $m I \leq X \leq M I$, then we have

$$
\begin{aligned}
\nu(1-\nu) \min \left\{1, m^{3}\right\} & \left(X^{-1}-I\right)^{2} \\
& \leq(1-\nu) I+\nu X-\left((1-\nu) I+\nu X^{-1}\right)^{-1} \\
& \leq \nu(1-\nu) \max \left\{1, M^{3}\right\}\left(X^{-1}-I\right)^{2}
\end{aligned}
$$

for any $\nu \in[0,1]$.

If we multiply (1.2) both sides by $A^{-1 / 2}$, we get $M I \geq A^{-1 / 2} B A^{-1 / 2} \geq$ $m I$.

By writing the inequality (2.10) for $X=A^{-1 / 2} B A^{-1 / 2}$, we obtain

$$
\begin{aligned}
& \nu(1-\nu) \min \left\{1, m^{3}\right\}\left(A^{1 / 2} B^{-1} A^{1 / 2}-I\right)^{2} \\
& \quad \leq(1-\nu) I+\nu A^{-1 / 2} B A^{-1 / 2}-\left((1-\nu) I+\nu A^{1 / 2} B^{-1} A^{1 / 2}\right)^{-1} \\
& \quad \leq \nu(1-\nu) \max \left\{1, M^{3}\right\}\left(A^{1 / 2} B^{-1} A^{1 / 2}-I\right)^{2}
\end{aligned}
$$

for any $\nu \in[0,1]$.

If we multiply both sides of the inequality $(2.11)$ by $A^{1 / 2}$, then we get

$$
\begin{aligned}
& \nu(1-\nu) \min \left\{1, m^{3}\right\} A^{1 / 2}\left(A^{1 / 2} B^{-1} A^{1 / 2}-I\right)^{2} A^{1 / 2} \\
& \quad \leq(1-\nu) A+\nu B-A^{1 / 2}\left(\nu A^{1 / 2} B^{-1} A^{1 / 2}+(1-\nu) I\right)^{-1} A^{1 / 2} \\
& \quad \leq \nu(1-\nu) \max \left\{1, M^{3}\right\} A^{1 / 2}\left(A^{1 / 2} B^{-1} A^{1 / 2}-I\right)^{2} A^{1 / 2}
\end{aligned}
$$

for any $\nu \in[0,1]$.

Observe that

$$
\begin{aligned}
A^{1 / 2} & \left(\nu\left(A^{-1 / 2} B A^{-1 / 2}\right)^{-1}+(1-\nu) I\right)^{-1} A^{1 / 2} \\
& =A^{1 / 2}\left(A^{1 / 2}\left(\nu B^{-1}+(1-\nu) A^{-1}\right) A^{1 / 2}\right)^{-1} A^{1 / 2} \\
& =A^{1 / 2} A^{-1 / 2}\left(\nu B^{-1}+(1-\nu) A^{-1}\right)^{-1} A^{-1 / 2} A^{1 / 2}=A{ }_{\nu} B
\end{aligned}
$$


and

$$
\begin{aligned}
A^{1 / 2} & \left(A^{1 / 2} B^{-1} A^{1 / 2}-I\right)^{2} A^{1 / 2} \\
& =A^{1 / 2} A^{1 / 2}\left(B^{-1}-A^{-1}\right) A^{1 / 2} A^{1 / 2}\left(B^{-1}-A^{-1}\right) A^{1 / 2} A^{1 / 2} \\
& =A\left(B^{-1}-A^{-1}\right) A\left(B^{-1}-A^{-1}\right) A \\
& =\left(A B^{-1}-I\right)^{2} A .
\end{aligned}
$$

From (2.12) we then get the desired result (2.1).

We define the weighted arithmetic and geometric means

$$
A_{\nu}(a, b):=(1-\nu) a+\nu b \text { and } G_{\nu}(a, b):=a^{1-\nu} b^{\nu},
$$

where $\nu \in[0,1]$ and $a, b>0$. If $\nu=\frac{1}{2}$, then we write for brevity $A(a, b)$ and $G(a, b)$, respectively.

Lemma 1. Let $M, m \in \mathbb{R}$ with $M>m$ and $\Phi: I \subseteq(0, \infty) \rightarrow \mathbb{R}$ be a twice differentiable function on I such that

$$
m \leq t^{2} \Phi^{\prime \prime}(t) \leq M,
$$

for any $t \in \stackrel{\circ}{I}$. Then for any $a, b \in \stackrel{\circ}{I}$ and $\nu \in[0,1]$ we have

$$
\begin{aligned}
m \ln \left(\frac{A_{\nu}(a, b)}{G_{\nu}(a, b)}\right) & \leq(1-\nu) \Phi(a)+\nu \Phi(b)-\Phi((1-\nu) a+\nu b) \\
& \leq M \ln \left(\frac{A_{\nu}(a, b)}{G_{\nu}(a, b)}\right) .
\end{aligned}
$$

In particular,

$$
m \ln \left(\frac{A(a, b)}{G(a, b)}\right) \leq \frac{\Phi(a)+\Phi(b)}{2}-\Phi\left(\frac{a+b}{2}\right) \leq M \ln \left(\frac{A(a, b)}{G(a, b)}\right) .
$$

Proof. The function $\Phi_{m}:=\Phi+m \ln$ is convex on $I$. Indeed, since $m \leq$ $t^{2} \Phi^{\prime \prime}(t)$ for any $t \in \stackrel{\circ}{I}$, we have

$$
\Phi_{m}^{\prime \prime}(t)=\Phi^{\prime \prime}(t)-\frac{m}{t^{2}}=\frac{\Phi^{\prime \prime}(t) t^{2}-m}{t^{2}} \geq 0, \quad t \in \stackrel{\circ}{I} .
$$


By the definition of convexity, we have

$$
\begin{aligned}
\Phi((1-\nu) a+\nu b) & +m \ln A_{\nu}(a, b) \\
& \leq(1-\nu)[\Phi(a)+m \ln a]+\nu[\Phi(b)+m \ln b] \\
& =(1-\nu) \Phi(a)+\nu \Phi(b)+(1-\nu) m \ln a+\nu m \ln b \\
& =(1-\nu) \Phi(a)+\nu \Phi(b)+m \ln G_{\nu}(a, b)
\end{aligned}
$$

for any $a, b \in \stackrel{\circ}{I}$ and $\nu \in[0,1]$, that is equivalent to

$$
m \ln \frac{A_{\nu}(a, b)}{G_{\nu}(a, b)} \leq(1-\nu) \Phi(a)+\nu \Phi(b)-\Phi((1-\nu) a+\nu b)
$$

for any $a, b \in \stackrel{\circ}{I}$ and $\nu \in[0,1]$ and the first inequality in (2.1) is proved.

Similarly, by the convexity of $\Phi_{M}:=-M \ln -\Phi$ we get the second part of $(2.1)$.

We recall that Specht's ratio is defined by

$$
S(h):= \begin{cases}\frac{h^{\frac{1}{h-1}}}{e \ln \left(h^{\frac{1}{h-1}}\right)} & \text { if } h \in(0,1) \cup(1, \infty), \\ 1 & \text { if } h=1 .\end{cases}
$$

It is well known that $\lim _{h \rightarrow 1} S(h)=1, S(h)=S\left(\frac{1}{h}\right)>1$ for $h>0, h \neq 1$. The function is decreasing on $(0,1)$ and increasing on $(1, \infty)$.

The following inequality provides a refinement and a multiplicative reverse for Young's inequality

$$
S\left(\left(\frac{a}{b}\right)^{r}\right) \leq \frac{A_{\nu}(a, b)}{G_{\nu}(a, b)} \leq S\left(\frac{a}{b}\right),
$$

where $a, b>0, \nu \in[0,1], r=\min \{1-\nu, \nu\}$.

The second inequality in (2.17) is due to Tominaga [11] while the first one is due to Furuichi [8].

Corollary 1. With the assumptions of Lemma 1 we have

$$
\begin{aligned}
m \ln S\left(\left(\frac{a}{b}\right)^{r}\right) & \leq(1-\nu) \Phi(a)+\nu \Phi(b)-\Phi((1-\nu) a+\nu b) \\
& \leq M \ln S\left(\frac{a}{b}\right)
\end{aligned}
$$

for any $a, b \in \stackrel{\circ}{I}$ and $\nu \in[0,1]$, where $r=\min \{1-\nu, \nu\}$.

In particular,

$$
m \ln S\left(\sqrt{\frac{a}{b}}\right) \leq \frac{\Phi(a)+\Phi(b)}{2}-\Phi\left(\frac{a+b}{2}\right) \leq M \ln S\left(\frac{a}{b}\right) .
$$

We consider Kantorovich's ratio defined by

$$
K(h):=\frac{(h+1)^{2}}{4 h}, \quad h>0 .
$$


The function $K$ is decreasing on $(0,1)$ and increasing on $[1, \infty), K(h) \geq 1$ for any $h>0$ and $K(h)=K\left(\frac{1}{h}\right)$ for any $h>0$.

The following multiplicative refinement and reverse of Young's inequality in terms of Kantorovich's ratio hold

$$
K^{r}\left(\frac{a}{b}\right) \leq \frac{A_{\nu}(a, b)}{G_{\nu}(a, b)} \leq K^{R}\left(\frac{a}{b}\right),
$$

where $a, b>0, \nu \in[0,1], r=\min \{1-\nu, \nu\}$ and $R=\max \{1-\nu, \nu\}$.

The first inequality in (2.21) was obtained by Zou et al. in [12] while the second by Liao et al. [10].

Corollary 2. With the assumptions of Lemma 1 we have

$$
\begin{aligned}
m r \ln K\left(\frac{a}{b}\right) & \leq(1-\nu) \Phi(a)+\nu \Phi(b)-\Phi((1-\nu) a+\nu b) \\
& \leq M R \ln K\left(\frac{a}{b}\right)
\end{aligned}
$$

for any $a, b \in \stackrel{\circ}{I}$ and $\nu \in[0,1]$, where $r=\min \{1-\nu, \nu\}$ and $R=\max \{1-\nu, \nu\}$.

In particular,

$$
\frac{1}{2} m \ln K\left(\frac{a}{b}\right) \leq \frac{\Phi(a)+\Phi(b)}{2}-\Phi\left(\frac{a+b}{2}\right) \leq \frac{1}{2} M \ln K\left(\frac{a}{b}\right) .
$$

In the recent paper [2] we obtained the following multiplicative reverse of Young's inequality

$$
(1 \leq) \frac{(1-\nu) a+\nu b}{a^{1-\nu} b^{\nu}} \leq \exp \left[4 \nu(1-\nu)\left(K\left(\frac{a}{b}\right)-1\right)\right],
$$

where $a, b>0, \nu \in[0,1]$.

Using this inequality, we can state:

Corollary 3. With the assumptions of Lemma 1 we have

$$
\begin{aligned}
(0 & \leq)(1-\nu) \Phi(a)+\nu \Phi(b)-\Phi((1-\nu) a+\nu b) \\
& \leq 4 M \nu(1-\nu)\left(K\left(\frac{a}{b}\right)-1\right)
\end{aligned}
$$

for any $a, b \in \stackrel{\circ}{I}$ and $\nu \in[0,1]$, where $K$ is Kantorovich's ratio.

In particular,

$$
\frac{\Phi(a)+\Phi(b)}{2}-\Phi\left(\frac{a+b}{2}\right) \leq M\left(K\left(\frac{a}{b}\right)-1\right) .
$$

In the recent paper [3] we established the following refinement and reverse of multiplicative Young's inequality:

$$
\begin{gathered}
\exp \left[\frac{1}{2} \nu(1-\nu)\left(1-\frac{\min \{a, b\}}{\max \{a, b\}}\right)^{2}\right] \leq \frac{(1-\nu) a+\nu b}{a^{1-\nu} b^{\nu}} \\
\leq \exp \left[\frac{1}{2} \nu(1-\nu)\left(\frac{\max \{a, b\}}{\min \{a, b\}}-1\right)^{2}\right]
\end{gathered}
$$


for any $a, b>0$ and $\nu \in[0,1]$.

Corollary 4. With the assumptions of Lemma 1 we have

$$
\begin{aligned}
\frac{1}{2} \nu(1-\nu) & m\left(1-\frac{\min \{a, b\}}{\max \{a, b\}}\right)^{2} \\
\leq & (1-\nu) \Phi(a)+\nu \Phi(b)-\Phi((1-\nu) a+\nu b) \\
\leq & \frac{1}{2} \nu(1-\nu) M\left(\frac{\max \{a, b\}}{\min \{a, b\}}-1\right)^{2}
\end{aligned}
$$

for any $a, b \in \stackrel{\circ}{I}$ and $\nu \in[0,1]$, where $K$ is Kantorovich's ratio.

In particular,

$$
\begin{aligned}
\frac{1}{8} m\left(1-\frac{\min \{a, b\}}{\max \{a, b\}}\right)^{2} & \leq \frac{\Phi(a)+\Phi(b)}{2}-\Phi\left(\frac{a+b}{2}\right) \\
& \leq \frac{1}{8} M\left(\frac{\max \{a, b\}}{\min \{a, b\}}-1\right)^{2} .
\end{aligned}
$$

We can state now the following result concerning upper and lower bounds for the difference between the weighted arithmetic and harmonic means:

Lemma 2. Let $a, b>0$, then we have

$$
\begin{aligned}
2 \min \{a, b\} \ln \left(\frac{G_{\nu}(a, b)}{H_{\nu}(a, b)}\right) & \leq A_{\nu}(a, b)-H_{\nu}(a, b) \\
& \leq 2 \max \{a, b\} \ln \left(\frac{G_{\nu}(a, b)}{H_{\nu}(a, b)}\right)
\end{aligned}
$$

for any $\nu \in[0,1]$, where $H_{\nu}(a, b):=\left((1-\nu) a^{-1}+\nu b^{-1}\right)^{-1}$ is the weighted harmonic mean.

In particular,

$$
\begin{aligned}
2 \min \{a, b\} \ln \left(\frac{G(a, b)}{H(a, b)}\right) & \leq A(a, b)-H(a, b) \\
& \leq 2 \max \{a, b\} \ln \left(\frac{G(a, b)}{H(a, b)}\right),
\end{aligned}
$$

where $H(a, b)=\frac{2 a b}{a+b}$ is the harmonic mean.

Proof. Let $x, y>0$ with $x \neq y$ and $t \in[\min \{x, y\}, \max \{x, y\}]$. Consider

$$
\Phi:[\min \{x, y\}, \max \{x, y\}] \rightarrow(0, \infty), \quad \Phi(t)=\frac{1}{t} .
$$

Then $\Phi^{\prime \prime}(t)=\frac{2}{t^{3}}$ and

for any $t \in[\min \{x, y\}, \max \{x, y\}]$.

$$
\frac{2}{\max \{x, y\}} \leq t^{2} \Phi^{\prime \prime}(t) \leq \frac{2}{\min \{x, y\}}
$$


Writing inequality (2.14) for the function $\Phi(t)=\frac{1}{t}$, we have

$$
\begin{aligned}
\frac{2}{\max \{x, y\}} \ln \left(\frac{A_{\nu}(x, y)}{G_{\nu}(x, y)}\right) & \leq(1-\nu) \frac{1}{x}+\nu \frac{1}{y}-((1-\nu) x+\nu y)^{-1} \\
& \leq \frac{2}{\min \{x, y\}} \ln \left(\frac{A_{\nu}(x, y)}{G_{\nu}(x, y)}\right)
\end{aligned}
$$

for any $x, y>0$ and $\nu \in[0,1]$.

Let $x=\frac{1}{a}, y=\frac{1}{b}$ with $a, b>0$. Then by (2.32) we get

$$
\begin{aligned}
\frac{2}{\max \left\{\frac{1}{a}, \frac{1}{b}\right\}} & \ln \left(\frac{A_{\nu}\left(\frac{1}{a}, \frac{1}{b}\right)}{G_{\nu}\left(\frac{1}{a}, \frac{1}{b}\right)}\right) \\
\leq & (1-\nu) a+\nu b-\left((1-\nu) \frac{1}{a}+\nu \frac{1}{b}\right)^{-1} \\
\leq & \frac{2}{\min \left\{\frac{1}{a}, \frac{1}{b}\right\}} \ln \left(\frac{A_{\nu}\left(\frac{1}{a}, \frac{1}{b}\right)}{G_{\nu}\left(\frac{1}{a}, \frac{1}{b}\right)}\right)
\end{aligned}
$$

for any $\nu \in[0,1]$, which is equivalent to the desired result, since

$$
\frac{A_{\nu}\left(\frac{1}{a}, \frac{1}{b}\right)}{G_{\nu}\left(\frac{1}{a}, \frac{1}{b}\right)}=\frac{G_{\nu}(a, b)}{H_{\nu}(a, b)} .
$$

By using (2.30), (2.17), (2.21), (2.24) and (2.27), we get

$$
\begin{aligned}
& 2 \min \{a, b\} \ln S\left(\left(\frac{b}{a}\right)^{r}\right) \leq A_{\nu}(a, b)-H_{\nu}(a, b) \\
& \leq 2 \max \{a, b\} \ln \left(S\left(\frac{b}{a}\right)\right), \\
& 2 r \min \{a, b\} \ln K\left(\frac{b}{a}\right) \leq A_{\nu}(a, b)-H_{\nu}(a, b) \\
& \leq 2 R \max \{a, b\} \ln K\left(\frac{b}{a}\right), \\
& A_{\nu}(a, b)-H_{\nu}(a, b) \leq 8 \max \{a, b\} \nu(1-\nu)\left(K\left(\frac{b}{a}\right)-1\right),
\end{aligned}
$$

and

$$
\begin{aligned}
\nu(1-\nu) \min \{a, b\} & \left(1-\frac{\min \{a, b\}}{\max \{a, b\}}\right)^{2} \leq A_{\nu}(a, b)-H_{\nu}(a, b) \\
& \leq \nu(1-\nu) \max \{a, b\}\left(\frac{\max \{a, b\}}{\min \{a, b\}}-1\right)^{2}
\end{aligned}
$$

for any $a, b>0$ and $\nu \in[0,1]$, where $r=\min \{1-\nu, \nu\}$ and $R=\max \{1-\nu, \nu\}$. 
We have the following upper and lower bounds in terms of Specht's ratio.

Theorem 3. Let $A, B$ be positive invertible operators and $M>m>0$ be such that the condition (1.2) is valid. Then for any $\nu \in[0,1]$ we have

$$
2 u_{\nu}(m, M) A \leq A \nabla_{\nu} B-A !_{\nu} B \leq 2 U(m, M) A,
$$

where

$$
u_{\nu}(m, M):= \begin{cases}m \ln S\left(M^{r}\right) & \text { if } M<1 \\ 0 & \text { if } m \leq 1 \leq M \\ \ln S\left(m^{r}\right) & \text { if } 1<m\end{cases}
$$

and

$$
U(m, M):= \begin{cases}\ln S(m) & \text { if } M<1, \\ M \max \{\ln S(m), \ln S(M)\} & \text { if } m \leq 1 \leq M, \\ M \ln S(M) & \text { if } 1<m .\end{cases}
$$

Proof. By taking $a=1$ and $b=x>0$ in (2.35) we get

$$
\begin{aligned}
2 \min \{1, x\} \ln S\left(x^{r}\right) & \leq 1-\nu+\nu x-\left(1-\nu+\nu x^{-1}\right)^{-1} \\
& \leq 2 \max \{1, x\} \ln (S(x)),
\end{aligned}
$$

for any $\nu \in[0,1]$.

If $x \in[m, M] \subset(0, \infty)$, then $\min \{1, x\} \geq \min \{1, m\}$ and $\max \{1, x\} \leq$ $\max \{1, M\}$. By using the inequality (2.40), we get

$$
\begin{aligned}
2 \min \{1, m\} \min _{x \in[m, M]} \ln S\left(x^{r}\right) & \leq 1-\nu+\nu x-\left(1-\nu+\nu x^{-1}\right)^{-1} \\
& \leq 2 \max \{1, M\} \max _{x \in[m, M]} \ln (S(x))
\end{aligned}
$$

for any $\nu \in[0,1]$.

If we use the continuous functional calculus for the positive invertible operator $X$ with $m I \leq X \leq M I$, then by (2.41) we have

$$
\begin{aligned}
2 \min \{1, m\} & \min _{x \in[m, M]} \ln S\left(x^{r}\right) I \\
\leq & (1-\nu) I+\nu X-\left((1-\nu) I+\nu X^{-1}\right)^{-1} \\
& \leq 2 \max \{1, M\} \max _{x \in[m, M]} \ln (S(x)) I
\end{aligned}
$$

for any $\nu \in[0,1]$.

Now, by a similar argument to the one from Theorem 2, we conclude that

$$
\begin{aligned}
2 \min \{1, m\} \min _{x \in[m, M]} \ln S\left(x^{r}\right) A & \leq A \nabla_{\nu} B-A !_{\nu} B \\
& \leq 2 \max \{1, M\} \max _{x \in[m, M]} \ln (S(x)) A
\end{aligned}
$$

for any $\nu \in[0,1]$. 
Now it is enough to observe that, by the properties of the Specht's ratio, we have

$$
\min \{1, m\} \min _{x \in[m, M]} \ln S\left(x^{r}\right)= \begin{cases}m \ln S\left(M^{r}\right) & \text { if } M<1, \\ 0 & \text { if } m \leq 1 \leq M, \\ \ln S\left(m^{r}\right) & \text { if } 1<m\end{cases}
$$

and

$$
\max \{1, M\} \max _{x \in[m, M]} \ln (S(x))= \begin{cases}\ln S(m) & \text { if } M<1, \\ M \max \{\ln S(m), \ln S(M)\} & \text { if } m \leq 1 \leq M, \\ M \ln S(M) & \text { if } 1<m .\end{cases}
$$

In particular, we have

$$
2 u(m, M) A \leq A \nabla B-A ! B \leq 2 U(m, M) A
$$

where

$$
u(m, M):= \begin{cases}m \ln S(\sqrt{M}) & \text { if } M<1, \\ 0 & \text { if } m \leq 1 \leq M, \\ \ln S(\sqrt{m}) & \text { if } 1<m .\end{cases}
$$

We have the following upper and lower bounds in terms of Kantorovich's ratio.

Theorem 4. Let $A, B$ be positive invertible operators and $M>m>0$ be such that the condition (1.2) is valid. Then for any $\nu \in[0,1]$ we have

$$
2 r v(m, M) A \leq A \nabla_{\nu} B-A{ }_{\nu} B \leq 2 R V(m, M) A,
$$

where

$$
v(m, M):= \begin{cases}m \ln K(M) & \text { if } M<1, \\ 0 & \text { if } m \leq 1 \leq M \\ \ln K(m) & \text { if } 1<m\end{cases}
$$

and

$$
V(m, M):= \begin{cases}\ln K(m) & \text { if } M<1, \\ M \max \{\ln K(m), \ln K(M)\} & \text { if } m \leq 1 \leq M \\ M \ln K(M) & \text { if } 1<m .\end{cases}
$$


We also have

$$
A \nabla_{\nu} B-A !_{\nu} B \leq 8 \nu(1-\nu) T(m, M) A,
$$

where

$$
T(m, M):= \begin{cases}K(m)-1 & \text { if } M<1, \\ M \max \{K(m)-1, K(M)-1\} & \text { if } m \leq 1 \leq M, \\ M(K(M)-1) & \text { if } 1<m .\end{cases}
$$

In particular, we have

$$
v(m, M) A \leq A \nabla B-A ! B \leq V(m, M) A
$$

and

$$
A \nabla B-A ! B \leq 2 T(m, M) A .
$$

The inequality (2.44) follows from (2.37) and the following formulas:

$$
\min \{1, m\} \min _{x \in[m, M]} \ln K(x)= \begin{cases}m \ln K(M) & \text { if } M<1, \\ 0 & \text { if } m \leq 1 \leq M, \\ \ln K(m) & \text { if } 1<m\end{cases}
$$

and

$$
\begin{aligned}
\max \{1, M\} & \max _{x \in[m, M]} \ln K(x) \\
& = \begin{cases}\ln K(m) & \text { if } M<1, \\
M \max \{\ln K(m), \ln K(M)\} & \text { if } m \leq 1 \leq M, \\
M \ln K(M) & \text { if } 1<m,\end{cases}
\end{aligned}
$$

which can be derived from the properties of Kantorovich's ratio.

The inequality (2.47) follows by (2.36) and the formulas:

$$
\begin{aligned}
\max \{1, M\} & \max _{x \in[m, M]}(K(x)-1) \\
& = \begin{cases}K(m)-1 & \text { if } M<1, \\
M \max \{K(m)-1, K(M)-1\} & \text { if } m \leq 1 \leq M, \\
M(K(M)-1) & \text { if } 1<m .\end{cases}
\end{aligned}
$$

Now, if we take in (2.38) $a=1$ and $b=x$, then we have

$$
\begin{aligned}
\nu(1-\nu) & \min \{1, x\}\left(1-\frac{\min \{1, x\}}{\max \{1, x\}}\right)^{2} \\
& \leq 1-\nu+\nu x-\left(1-\nu+\nu x^{-1}\right)^{-1} \\
& \leq \nu(1-\nu) \max \{1, x\}\left(\frac{\max \{1, x\}}{\min \{1, x\}}-1\right)^{2} .
\end{aligned}
$$


If $x \in[m, M] \subset(0, \infty)$, then $\min \{1, x\} \geq \min \{1, m\}$ and $\max \{1, x\} \leq$ $\max \{1, M\}$. From (2.51) we then have

$$
\begin{aligned}
\nu(1-\nu) & \min \{1, m\}\left(1-\frac{\min \{1, m\}}{\max \{1, M\}}\right)^{2} \\
& \leq 1-\nu+\nu x-\left(1-\nu+\nu x^{-1}\right)^{-1} \\
& \leq \nu(1-\nu) \max \{1, M\}\left(\frac{\max \{1, M\}}{\min \{1, m\}}-1\right)^{2}
\end{aligned}
$$

for any $x \in[m, M]$.

Using (2.52), we obtain the following operator inequality:

Theorem 5. Let $A, B$ be positive invertible operators and $M>m>0$ be such that the condition (1.2) is valid. Then for any $\nu \in[0,1]$ we have

$$
\nu(1-\nu) z(m, M) A \leq A \nabla_{\nu} B-A !_{\nu} B \leq \nu(1-\nu) Z(m, M) A,
$$

where

$$
z(m, M):= \begin{cases}m(1-m)^{2} & \text { if } M<1, \\ m\left(1-\frac{m}{M}\right)^{2} & \text { if } m \leq 1 \leq M, \\ \left(1-\frac{1}{M}\right)^{2} & \text { if } 1<m\end{cases}
$$

and

$$
Z(m, M):= \begin{cases}\left(\frac{1}{m}-1\right)^{2} & \text { if } M<1, \\ M\left(\frac{M}{m}-1\right)^{2} & \text { if } m \leq 1 \leq M, \\ M(M-1)^{2} & \text { if } 1<m .\end{cases}
$$

In particular,

$$
\frac{1}{4} z(m, M) A \leq A \nabla B-A ! B \leq \frac{1}{4} Z(m, M) A .
$$

3. Applications. We apply some of the above results for operators that are bounded below and above by positive constants.

Proposition 1. Let $A, B$ be two positive operators and $m, m^{\prime}, M, M^{\prime}$ be positive real numbers. Put $h:=\frac{M}{m}$ and $h^{\prime}:=\frac{M^{\prime}}{m^{\prime}}$.

(i) If $0<m I \leq A \leq m^{\prime} I<M^{\prime} I \leq B \leq M I$, then

$$
\begin{aligned}
\nu(1-\nu)\left(A B^{-1}-I\right)^{2} A & \leq A \nabla_{\nu} B-A !_{\nu} B \\
& \leq \nu(1-\nu) h^{3}\left(A B^{-1}-I\right)^{2} A .
\end{aligned}
$$

(ii) If $0<m I \leq B \leq m^{\prime} I<M^{\prime} I \leq A \leq M I$, then

$$
\begin{aligned}
\nu(1-\nu) \frac{1}{h^{3}}\left(A B^{-1}-I\right)^{2} A & \leq A \nabla_{\nu} B-A !_{\nu} B \\
& \leq \nu(1-\nu)\left(A B^{-1}-I\right)^{2} A .
\end{aligned}
$$


Proof. We observe that $h, h^{\prime}>1$ and if either of the condition (i) or (ii) holds, then $h \geq h^{\prime}$.

If (i) is valid, then we have

$$
A<h^{\prime} A=\frac{M^{\prime}}{m^{\prime}} A \leq B \leq \frac{M}{m} A=h A,
$$

while, if (ii) is valid, then we have

$$
\frac{1}{h} A \leq B \leq \frac{1}{h^{\prime}} A<A .
$$

If we use the inequality (2.1) and the assumption (i), then we get (3.1).

If we use the inequality (2.1) and the assumption (ii), then we get (3.2).

The following result provides bounds in terms of Specht's ratio.

Proposition 2. Let $A, B$ be two positive operators and $m, m^{\prime}, M, M^{\prime}$ be positive real numbers. Put $h:=\frac{M}{m}$ and $h^{\prime}:=\frac{M^{\prime}}{m^{\prime}}$.

(i) If $0<m I \leq A \leq m^{\prime} I<M^{\prime} I \leq B \leq M I$, then

$$
2 \ln S\left(\left(h^{\prime}\right)^{r}\right) A \leq A \nabla_{\nu} B-A !_{\nu} B \leq 2 h \ln S(h) A .
$$

(ii) If $0<m I \leq B \leq m^{\prime} I<M^{\prime} I \leq A \leq M I$, then

$$
2 \frac{1}{h} \ln S\left(\left(h^{\prime}\right)^{r}\right) A \leq A \nabla_{\nu} B-A !_{\nu} B \leq 2 \ln S(h) A .
$$

Proof. If we use the inequality (2.39) and the assumption (i), then we have

$$
2 \ln S\left(\left(h^{\prime}\right)^{r}\right) A \leq A \nabla_{\nu} B-A !_{\nu} B \leq 2 h \ln S(h) A
$$

and the inequality (3.5) is proved.

If we use the assumption (ii) and the inequality (2.39), then we get

$$
2 \frac{1}{h} \ln S\left(\left(\frac{1}{h^{\prime}}\right)^{r}\right) A \leq A \nabla_{\nu} B-A !_{\nu} B \leq 2 \ln S\left(\frac{1}{h}\right) A .
$$

Since $S\left(\left(\frac{1}{h^{\prime}}\right)^{r}\right)=S\left(\left(h^{\prime}\right)^{r}\right)$ and $S\left(\frac{1}{h}\right)=S(h)$ then by (3.8) we get (3.6).

We also have upper and lower bounds in terms of Kantorovich's ratio:

Proposition 3. With the assumptions of Proposition 2 we have:

(i) If $0<m I \leq A \leq m^{\prime} I<M^{\prime} I \leq B \leq M I$, then

$$
2 r \ln K\left(h^{\prime}\right) A \leq A \nabla_{\nu} B-A !_{\nu} B \leq 2 R h \ln K(h) A .
$$

(ii) If $0<m I \leq B \leq m^{\prime} I<M^{\prime} I \leq A \leq M I$, then

$$
2 r \frac{1}{h} \ln K\left(h^{\prime}\right) A \leq A \nabla_{\nu} B-A !_{\nu} B \leq 2 R \ln K(h) A .
$$


Proof. Using the inequality (2.44) and the assumption (i), we have

$$
2 r \ln K\left(h^{\prime}\right) A \leq A \nabla_{\nu} B-A !_{\nu} B \leq 2 R h \ln K(h) A,
$$

and the inequality (3.9) is proved.

By the assumptions (ii) and the inequality (2.44) we also have

$$
2 r \frac{1}{h} \ln K\left(\frac{1}{h^{\prime}}\right) A \leq A \nabla_{\nu} B-A !_{\nu} B \leq 2 R \ln K\left(\frac{1}{h}\right) A,
$$

and since $K\left(\frac{1}{h^{\prime}}\right)=K\left(h^{\prime}\right)$ and $K\left(\frac{1}{h}\right)=K(h)$, we deduce the desired result (3.10).

We finally have:

Proposition 4. With the assumptions of Proposition 2 we have:

(i) If $0<m I \leq A \leq m^{\prime} I<M^{\prime} I \leq B \leq M I$, then

$$
\nu(1-\nu)\left(1-\frac{1}{h}\right)^{2} A \leq A \nabla_{\nu} B-A !_{\nu} B \leq \nu(1-\nu) h(h-1)^{2} A .
$$

(ii) If $0<m I \leq B \leq m^{\prime} I<M^{\prime} I \leq A \leq M I$, then

$$
\nu(1-\nu) \frac{1}{h}\left(1-\frac{1}{h}\right)^{2} A \leq A \nabla_{\nu} B-A !_{\nu} B \leq \nu(1-\nu)(h-1)^{2} A .
$$

The proof follows by (2.53) and we omit the details.

Now, if we consider the following two variable functions obtained by taking the upper bounds for the difference $A_{\nu}(a, b)-H_{\nu}(a, b)$ given by the inequalities (2.35)-(2.38) for $a=1, b=x \in(0, \infty)$ and $y \in(0,1)$, namely

$$
\begin{aligned}
& U_{1}(x, y):=2 \max \{x, 1\} \ln S(x), \\
& U_{2}(x, y):=2 \max \{y, 1-y\} \max \{x, 1\} \ln K(x), \\
& U_{3}(x, y):=8 y(1-y) \max \{x, 1\}(K(x)-1), \\
& U_{4}(x, y):=y(1-y) \max \{x, 1\}\left(\frac{\max \{x, 1\}}{\min \{x, 1\}}-1\right)^{2},
\end{aligned}
$$

then the differences $U_{1}-U_{2}, U_{1}-U_{3}, U_{1}-U_{4}, U_{2}-U_{3}, U_{2}-U_{4}$ take both negative and positive values on the box $(0,10) \times(0,1)$, showing that neither of these bounds are best in general. However, the plot of the difference $U_{3}-U_{4}$ takes only negative values on the box $(0,10) \times(0,1)$, suggesting that the upper bound in (2.38) may be better than that in (2.37). It is an open question for the author if this is true in general.

Acknowledgement. The author would like to thank the anonymous referee for valuable suggestions that have been implemented in the final version of the paper. 


\section{REFERENCES}

[1] Dragomir, S. S., Bounds for the normalized Jensen functional, Bull. Austral. Math. Soc. 74 (3) (2006), 417-478.

[2] Dragomir, S. S., A note on Young's inequality, Rev. R. Acad. Cienc. Exactas Fís. Nat. Ser. A Mat. RACSAM 111 (2) (2017), 349-354.

[3] Dragomir, S. S., Some new reverses of Young's operator inequality, Preprint RGMIA Res. Rep. Coll. 18 (2015), Art. 130. http://rgmia.org/papers/v18/v18a130.pdf

[4] Dragomir, S. S., On new refinements and reverses of Young's operator inequality, Transylv. J. Math. Mech. 8 (1) (2016), 45-49.

[5] Dragomir, S. S., Some inequalities for operator weighted geometric mean, Preprint RGMIA Res. Rep. Coll. 18 (2015), Art. 139. http://rgmia.org/papers/v18/v18a139.pdf

[6] Dragomir, S. S., Some reverses and a refinement of Hölder operator inequality, Preprint RGMIA Res. Rep. Coll. 18 (2015), Art. 147. http://rgmia.org/papers/v18/v18a147.pdf

[7] Dragomir, S. S., Some inequalities for weighted harmonic and arithmetic operator means, Fasc. Math. No. 61 (2018), 43-54.

[8] Furuichi, S., Refined Young inequalities with Specht's ratio, J. Egyptian Math. Soc. 20 (2012), 46-49.

[9] Furuichi, S., On refined Young inequalities and reverse inequalities, J. Math. Inequal. 5 (2011), 21-31.

[10] Liao, W., Wu, J., Zhao, J., New versions of reverse Young and Heinz mean inequalities with the Kantorovich constant, Taiwanese J. Math. 19 (2) (2015), 467-479.

[11] Tominaga, M., Specht's ratio in the Young inequality, Sci. Math. Japon. 55 (2002), $583-588$.

[12] Zuo, G., Shi, G., Fujii, M., Refined Young inequality with Kantorovich constant, J. Math. Inequal. 5 (2011), 551-556.

\section{S. S. Dragomir}

College of Engineering \& Science

Victoria University, PO Box 14428

Melbourne City, MC 8001, Australia

e-mail: sever.dragomir@vu.edu . au

School of Computer Science \& Applied Mathematics University of the Witwatersrand, Private Bag 3

Johannesburg 2050, South Africa

Received November 20, 2018 